\title{
ARTICLE
}

Epidemiology

\section{Real-world data on cervical cancer risk stratification by cytology and HPV genotype to inform the management of HPV-positive women in routine cervical screening}

\author{
Dana Hashim ${ }^{1}$, Birgit Engesæter ${ }^{1}$, Gry Baadstrand Skare ${ }^{1}$, Philip E. Castle ${ }^{2}$, Tone Bjørge $\mathbb{I D}^{1,3}$, Ameli Tropé ${ }^{1}$ and Mari Nygård $\mathbb{D}^{4}$
}

\begin{abstract}
BACKGROUND: HPV16/18 detection may improve cervical cancer risk stratification and better guide which HPV-positive women warrant immediate colposcopy/biopsy. We estimated risks of cervical precancer and cancer by HPV genotype and cytology during the implementation phase of primary HPV testing in Norway.

METHODS: A total of 3111 women, aged 34-69 years, testing HPV-positive at baseline and undergoing cytology testing from February 2015 to April 2018 had data available for analysis. Risk estimates with $95 \%$ confidence intervals (95\%Cls) of cervical intraepithelial neoplasia grade 3 or more severe (CIN3+) were estimated for cytology results and HPV genotypes (HPV16, HPV18, and other high-risk HPV).

RESULTS: CIN3 + risks were higher for HPV16/18 than other high-risk HPV genotypes. Among women with any cytologic abnormality [atypical squamous cells of undetermined significance or worse], immediate risks were $57.8 \%(95 \% \mathrm{Cl}=53.0-62.6 \%)$ for HPV16, 40.2\% (95\%Cl = 32.3-49.2\%) for HPV18, and 31.4\% $(95 \% \mathrm{Cl}=28.7-34.3 \%)$ for other high-risk HPV. Among those with normal cytology, CIN3 + risks were $19.9 \%(95 \% \mathrm{Cl}=15.0-26.1 \%)$ for HPV16 positives, $10.8 \%(95 \% \mathrm{Cl}=5.6-20.5 \%)$ for HPV18 positives, and $5.5 \%(95 \% \mathrm{Cl}=4.2-7.1 \%)$ for other high-risk HPV.

CONCLUSIONS: The benefits and harms of managing women based on HPV positivity and cytology results can be better balanced by inclusion of HPV genotyping in screening and choosing more conservative management for other high-risk HPV compared to HPV16/18.
\end{abstract}

British Journal of Cancer (2020) 122:1715-1723; https://doi.org/10.1038/s41416-020-0790-1

\section{BACKGROUND}

Women with human papillomavirus (HPV) infections that persist for at least one ${ }^{1}$ or two years ${ }^{2}$ are at high risk of cervical precancers (cervical intraepithelial neoplasia grade 2 [CIN2], grade 3 [CIN3], adenocarcinoma in situ [AIS] and cervical cancer). HPV infection in women over 30 years old is more likely to be persistent, with a greater likelihood of the development of highgrade cervical intraepithelial neoplasia (CIN2/3) or cervical cancer. ${ }^{3}$

Randomised controlled trials have demonstrated that primary HPV screening leads to a greater reduction in the overall incidence of cervical cancer compared to conventional cytology-based screening and it is now recommended to use HPV as a screening test for women over 30 years of age. ${ }^{4,5}$ HPV testing is also more sensitive than cervical cytology alone in detecting CIN2 or more severe diagnoses $(\mathrm{CIN} 2+)$ and $\mathrm{CIN} 3$ or more severe diagnoses $(\mathrm{CIN} 3+) .{ }^{5-8}$ However, primary HPV screening also detects transient HPV infections, which increases the number of positive screening results and potentially the number of colposcopies performed, ${ }^{5,9}$ the latter depending on the criteria for referral to colposcopy.

Particularly for HPV-positive women with cytology negative for intraepithelial lesion or malignancy (NILM) or low-grade cytology, over-referral to colposcopy can lead to a larger number of colposcopy/biopsy results negative for CIN2.$+{ }^{10}$ This presents a major challenge to the limited colposcopy capacity in most healthcare systems. ${ }^{10,11}$ It is important to choose a colposcopy referral threshold based on the balance between benefits and harms.

The optimal triage protocol for HPV-positive women in routine clinical setting is not yet determined but cytology is currently the general standard. The risk of $\mathrm{CIN} 2+$ and $\mathrm{CIN} 3+$ varies among oncogenic HPV genotypes., ${ }^{2-15}$ HPV16 and/or 18 infection are found in $70 \%$ of cervical cancers, ${ }^{16}$ and confer a higher risk than other high-risk HPV genotypes. ${ }^{17,18}$ Among women undergoing primary HPV screening, partial HPV genotyping in combination with cytology may better determine women who should be referred to biopsy compared to cytology alone. In a large US cohort study, women positive for HPV16 were at highest risk for $\mathrm{CIN} 3+$, followed by HPV18. ${ }^{19}$ Similar results were also found in a Japanese longitudinal study ${ }^{20}$ and a large clinical trial, ATHENA (Addressing the Need for Advanced HPV Diagnosis). ${ }^{21}$ However, observational studies and randomised controlled trials have specific enrolment criteria that may limit the applicability of results to whole populations. Such limitations include results reflecting subgroups of populations and possible under- or over-

\footnotetext{
${ }^{1}$ Section for cervical cancer screening, Cancer Registry of Norway, Oslo, Norway; ${ }^{2}$ Department of Epidemiology and Population Health, Albert Einstein College of Medicine, Bronx, NY, USA; ${ }^{3}$ Department of Global Public Health and Primary Care, University of Bergen, Bergen, Norway and ${ }^{4}$ Department of Research, Cancer Registry of Norway, Oslo, Norway Correspondence: Mari Nygård (mari.nygard@kreftregisteret.no)
}

Received: 30 August 2019 Revised: 3 February 2020 Accepted: 12 February 2020

Published online: 3 April 2020 
estimation of outcomes due to censoring and aggressive followup. $^{21,22}$ Risk assessments using real-world or population-based data is needed to ensure "equal management for equal risk" for referral to colposcopy for populations with different patterns of care, compliance, testing, rescreening intervals, and risk factors. ${ }^{12,23}$ Consequently, national screening programs should evaluate and calibrate their screening algorithms based on accepted cervical cancer risk threshold for referring to colposcopy and based on the population's real-world screening data and capacity.

The Norwegian Cervical Cancer Screening Program (NCCSP) requires data generated within Norway using a real-world population base to modify national screening guidelines. This study aimed to estimate CIN3 + and CIN2+ risks by combinations of HPV16, HPV18, and other high-risk genotypes and cytological results among women in the Norwegian cervical cancer screening population.

\section{METHODS}

\section{Participants}

Women aged 34-69 years and living in four Norwegian counties (Sør- and Nord-Trøndelag, Hordaland and Rogaland), were pseudo-randomly assigned by birthday to undergo 5-yearly primary HPV screening or continue to receive 3-yearly cytology screening (even or odd day of birth, respectively), from February
2015 until April 2018. ${ }^{24}$ An exfoliated cervical specimen was collected during pelvic examination using a routinely available collection devices, usually a dry brush for collecting exfoliating cells, which was then rinsed immediately after collection into a ThinPrep (PreservCyt ${ }^{\circledR}$ Solution, Hologic, Inc., Marlborough, MA, USA) for HPV and liquid-based cytology (LBC) testing. Vials were then transported to one of three laboratories for pre-processing and evaluation. All participating laboratories followed a set of guidelines, approved by the Academic Panel for HPV screening implementation in Norway and described in the Quality Assurance Manual for the NCCSP, for allocation, conduction and follow-up of both screening strategies to unify implementation routines.

\section{Laboratory testing}

HPV testing. HPV testing was conducted utilising the cobas 4800 HPV test (Roche Diagnostics, Basel, Switzerland) as previously described. ${ }^{24}$ Briefly, the real-time polymerase chain reaction-based method detects 14 HPV genotypes. Results are reported separately for HPV16 and HPV18. The other 12 types (31, 33, 35, $39,45,51,52,56,58,59,66$, and 68 ) are reported concurrently as a pooled result referred to as other high-risk HPV. ${ }^{25,26}$

Cytology testing. For HPV-positive women, a baseline cytological evaluation of the cervical sample was performed at $T_{0}$, the beginning of the observation period for each subject (Supplementary Fig. 1). Liquid-based cytology was reported according

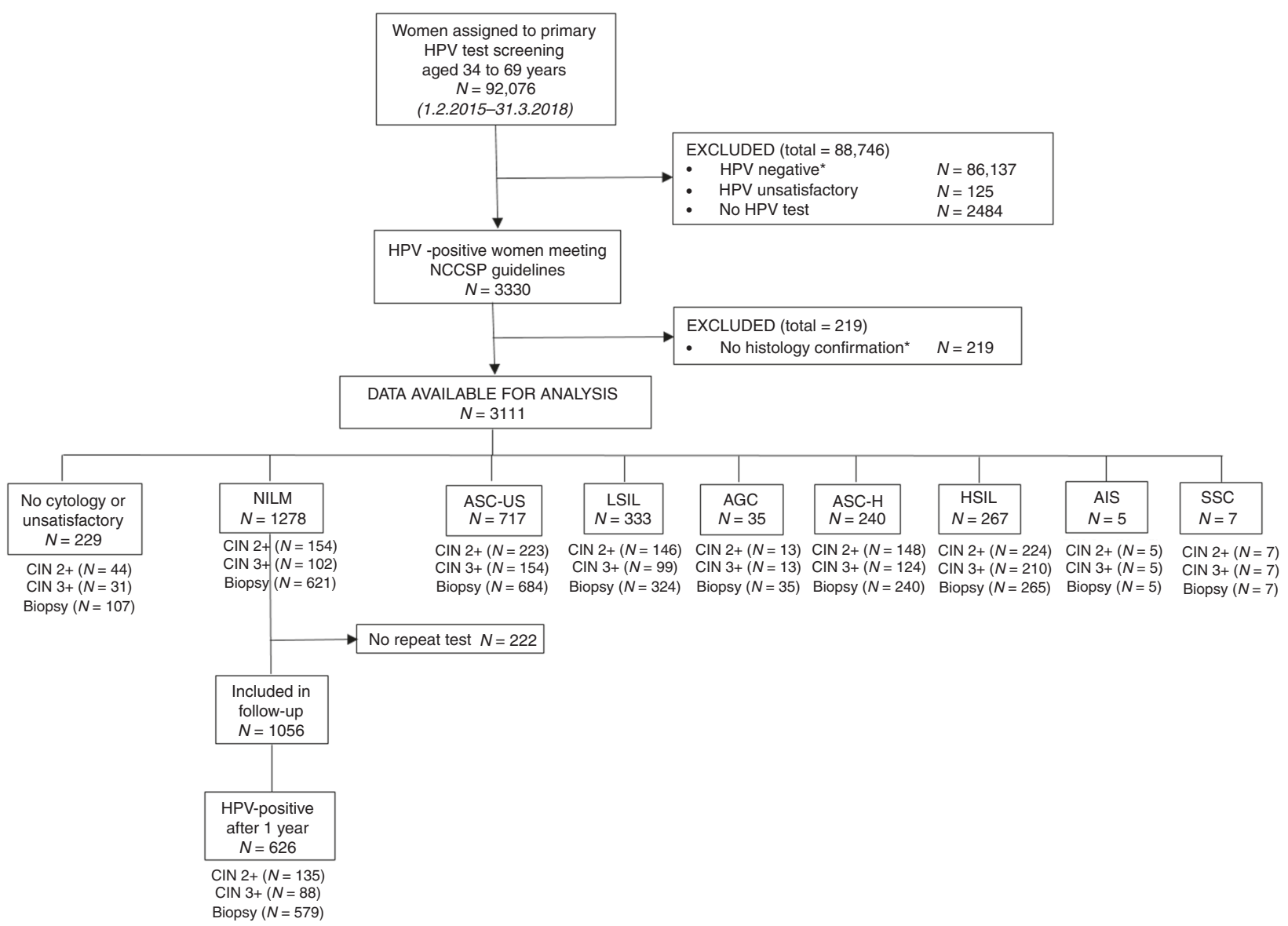

Fig. 1 Flow diagram describing selection criteria of primary HPV test participants in the Norwegian Cervical Cancer Screening Program, 2015 to 2018. *HPV negatives $(83,601)$ and those 2536 HPV positives with limited follow-up due to late testing because they had been screened towards the end of the enrolment period. Abbreviations: NILM, negative for intraepithelial lesion or malignancy; ASC-US, atypical squamous cells of undetermined significance; LSIL, low-grade squamous intraepithelial lesions, AGC, atypical glandular cells; ASC-H, atypical squamous cells, cannot rule out high-grade squamous intraepithelial lesion; HSIL, high-grade squamous intraepithelial lesions; ACIS; atypical glandular cells cervical adenocarcinoma in situ; SCC, squamous cell carcinoma. 
Real-world data on cervical cancer risk stratification by cytology and... D Hashim et al.

to the Bethesda system ${ }^{27}$ as unsatisfactory, NILM, atypical squamous cells of undetermined significance (ASC-US), lowgrade squamous intraepithelial lesion (LSIL), atypical glandular cells of undetermined significance (AGC), atypical squamous cells-cannot exclude HSIL (ASC-H), high-grade squamous intraepithelial lesion (HSIL), adenocarcinoma in situ (AIS) and cervical carcinoma. If the cytology result was ASC-US or more severe interpretation, "immediate" colposcopy and biopsy were recommended.

If the baseline cytology test was NILM, then a 12-month follow-up period was recommended prior to repeating the HPV testing. If the repeat HPV test was positive, women were referred for colposcopy with biopsy. If the repeat HPV test was negative, then women return to routine screening and scheduled to be rescreened by HPV testing in 5 years. ${ }^{28}$

A unique identification number, allowing identification of every legal resident in Norway, was used to link the information on cytology, HPV tests and histology at an individual level. Relevant screening results were extracted from the databases at the Cancer Registry of Norway for 92,076 women aged 34-69 years who underwent primary HPV screening with no recorded abnormal HPV-tests, cytology or histology (CIN2+) the last two years before the baseline screening test (Fig. 1). A total of 5866 (6.4\%) were HPV positive, although 2536 had been excluded due to late testing because they had been screened towards the end of the enrolment period. Exclusions were applied to women with unsatisfactory HPV test results with no repeat HPV test, HPVnegative results, and women with no histological confirmation. HPV negative women were excluded mainly because they require 5 years of follow up and the randomised implementation of HPV primary screening began in 2015. A total of 3111 HPVpositive women had complete data available for analysis that met NCCSP guidelines.

Use of Norwegian Cancer Registry data for identification and follow-up of study subjects

The Cancer Registry of Norway receives reports of cancer and precancerous lesions with compulsory central registration of cervical cytology, histology, and all HPV test results (both positive and negative), as previously described. ${ }^{29-31}$ Histological diagnoses endpoints were CIN2+ and CIN3+ according to WHO guidelines. ${ }^{32}$

Statistical analysis

Follow-up started at the date of the baseline cytology test and continued for all women until the worst histologically confirmed lesion was identified. All other observations were censored after the end of recommended follow up (9 months for HPV-positive and ASC-US or worse results or 21 months for HPV-positive and NILM results), which was according to NCCSP guidelines (Supplementary Fig. 1).

Cytology results of AGC, ASC-H, HSIL, AIS and cervical carcinoma were combined into a single "high-grade" category and LSIL and ASC-US were combined into a single "low-grade" category. HPV genotype results were categorised hierarchically according to cancer risk from highest to lowest risk: HPV16 > HPV18 > other high-risk HPV. ${ }^{33-36}$

Given that cervical carcinoma has a low incidence in Norway and the primary goal of cervical screening is to prevent invasive cancer, we used CIN3+ risk as our primary endpoint. We emphasised results for $\mathrm{CIN} 3+$ risk rather than CIN2+ because CIN2 is unreliably determined by pathologists ${ }^{37,38}$ and often regresses. $^{39,40}$

CIN3 + and CIN2 + risks with 95\% confidence intervals $(95 \% \mathrm{Cl})$ for each screening HPV genotype/cytology result combination were estimated using the Kaplan-Meier failure function. ${ }^{41}$ Risks of $\mathrm{CIN} 2+$ and $\mathrm{CIN} 3+$ were estimated among women with HPVpositive and ASC-US+ at 9 months and among women with HPVpositive and NILM at 21 months i.e. the baseline CIN3+ $\left(T_{0}\right)$ for
Table 1. Primary HPV test by cervical cytology and age group.

Total (\%) 34-43 years $44-69$ years

HPV positive ${ }^{a}$

Total $^{\mathrm{a}}$

No cytology or

unsatisfactory

NILM

Had repeat HPV test ${ }^{\mathrm{b}}$

ASC-US

LSIL

High-grade $^{c}$

$111+100+1507$

1604

16

Total

No cytology or

unsatisfactory

NILM

Had repeat HPV test ${ }^{b}$

ASC-US

LSIL

High-grade ${ }^{c}$

$\begin{array}{lll}229 & 7.4 & 120\end{array}$

109

$\begin{array}{lll}1278 & 41.1 \quad 545\end{array}$

733

$\begin{array}{llll}626 & 49.0^{\mathrm{b}} & 262\end{array}$

364

$\begin{array}{lll}717 & 23.0 & 329\end{array}$

388

143

$\begin{array}{lll}333 & 10.7 & 190\end{array}$

231

HPV 18

Total

$\begin{array}{lll}650 & 100 \quad 365\end{array}$

285

No cytology or

unsatisfactory

NILM

Had repeat HPV test ${ }^{\mathrm{b}}$

ASC-US

LSIL

High-grade ${ }^{c}$

HPV other high risk ${ }^{d}$

Total

No cytology or

unsatisfactory

NILM

Had repeat HPV test ${ }^{\mathrm{b}}$

ASC-US

LSIL

High-grade ${ }^{c}$

$\begin{array}{lll}49 & 7.5 \quad 25\end{array}$

24

$\begin{array}{llll}201 & 30.9 & 99 & 102\end{array}$

$116 \quad 57.7^{\mathrm{b}} \quad 63$

$\begin{array}{lll}132 & 20.3 & 67\end{array}$

$\begin{array}{lll}79 & 12.2 & 55\end{array}$

$\begin{array}{lll}189 & 29.1 \quad 119\end{array}$

53

65

24

70

102

$\begin{array}{llll}20 & 9.0 & 13 & 7\end{array}$

41

24

24

11

19

1173

73

578

282

284

105

133

CIN cervical intraepithelial neoplasia, NILM negative for intraepithelial lesion or malignancy, ASC-US atypical squamous cells of undetermined significance, $L S I L$ low grade squamous intraepithelial lesions.

${ }^{\text {a}}$ Total includes $92(3.0 \%)$ women who were not genotyped.

${ }^{b}$ Percentage is calculated as percent of those with NILM test who were positive upon repeat HPV test.

Includes AGC, ASC-H, HSIL, and cancer.

Includes HPV types 31, 33, 35, 39, 45, 51, 52, 56, 58, 59, 66, and 68.

those referred immediately to colposcopy and the subsequent CIN3+ risk for those undergoing follow-up HPV testing for a second time after 12 months, as per NCCSP. In the follow-up after 12 months, 222 (17\%) out of 1278 HPV-positive and NILM women did not come to the second round of screening. We also compared risks for younger (35-43 years) and older (44-69 years) women stratified by the median age of the screened women, using the log-rank test of equality for failure functions to test for significant differences by age. ${ }^{42}$ All statistical analyses were done using STATA statistical software: release 15 (College Station, TX: StataCorp LLC). 
Table 2. Risk of CIN3+ and CIN2+ among HPV-positive women by $\geq$ ASC-US cytology and HPV genotype.

\begin{tabular}{|c|c|c|c|c|c|c|c|c|c|}
\hline & Total & \multicolumn{4}{|l|}{ CIN3+ } & \multicolumn{4}{|l|}{ CIN 2+ } \\
\hline \multicolumn{10}{|l|}{ All HPV positive ${ }^{a}$} \\
\hline ASC-US & 717 & 154 & 21.5 & 18.7 & 24.7 & 223 & 31.1 & 27.9 & 34.6 \\
\hline LSIL & 333 & 99 & 29.7 & 25.2 & 35.0 & 146 & 43.8 & 38.7 & 49.4 \\
\hline High-grade $^{b}$ & 554 & 359 & 64.8 & 60.9 & 68.8 & 397 & 71.7 & 67.9 & 75.4 \\
\hline ASC-US & 132 & 51 & 38.6 & 30.9 & 47.5 & 63 & 47.7 & 39.6 & 56.6 \\
\hline LSIL & 79 & 37 & 46.8 & 36.6 & 58.4 & 49 & 62.0 & 51.5 & 72.6 \\
\hline High-grade & 189 & 143 & 75.7 & 69.4 & 81.5 & 153 & 81.0 & 75.1 & 86.2 \\
\hline$\geq$ ASC-US & 400 & 231 & 57.8 & 53.0 & 62.6 & 265 & 66.3 & 61.6 & 70.9 \\
\hline \multicolumn{10}{|l|}{ HPV18 } \\
\hline$\geq$ ASC-US & 127 & 51 & 40.2 & 32.3 & 49.2 & 67 & 52.8 & 44.4 & 61.6 \\
\hline \multicolumn{10}{|c|}{ Other high-risk HPV } \\
\hline ASC-US & 498 & 90 & 18.1 & 15.0 & 21.7 & 138 & 27.7 & 24.0 & 31.9 \\
\hline LSIL & 225 & 55 & 24.4 & 19.4 & 30.6 & 84 & 37.3 & 31.4 & 44.0 \\
\hline High-grade & 294 & 174 & 59.2 & 53.6 & 64.8 & 198 & 67.4 & 62.0 & 72.6 \\
\hline$\geq$ ASC-US & 1017 & 319 & 31.4 & 28.7 & 34.3 & 420 & 41.3 & 38.4 & 44.4 \\
\hline
\end{tabular}

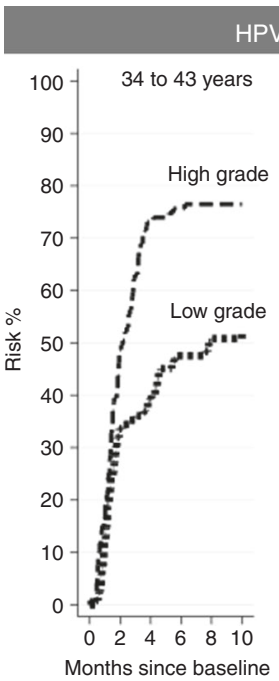

b

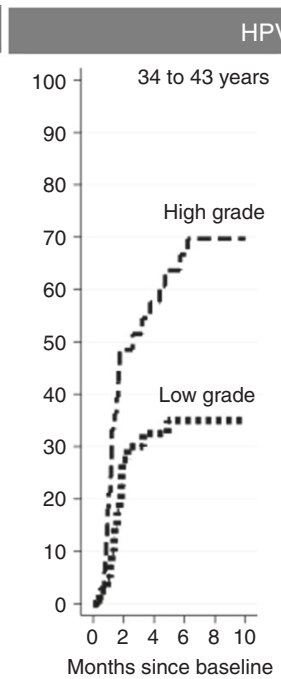

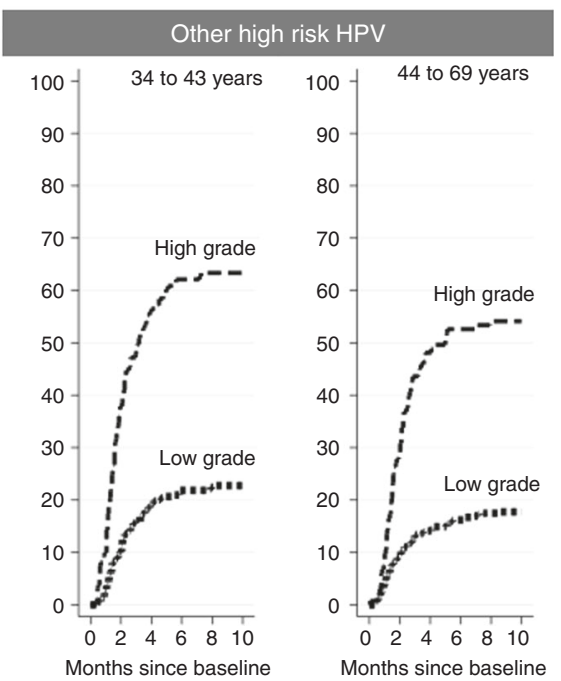

Fig. 2 Risk of CIN3 + by low-grade and high-grade cytology for 34-43 and 44-69 years old women by HPV genotype. (a) HPV16 genotype, (b) HPV18 genotype, (c) other high-risk HPV genotype. High grade: AGC, ASC-H, HSIL, ACIS, and SCC; Low grade: ASC-US and LSIL; Other highrisk HPV genotype includes 31, 33, 35, 39, 45, 51, 52, 56, 58, 59, 66, 68. Abbreviations: ASC-US, atypical squamous cells of undetermined significance; LSIL, low-grade squamous intraepithelial lesions, AGC, atypical glandular cells; ASC-H, atypical squamous cells, cannot rule out high-grade squamous intraepithelial lesion; HSIL, high-grade squamous intraepithelial lesions; ACIS; atypical glandular cells Cervical adenocarcinoma in situ; SCC, squamous cell carcinoma. 
Table 3. Risk of CIN3 + and CIN2+ among HPV-positive women with NILM cytology by HPV genotype at baseline screening until regular follow-up of 21 months.

\begin{tabular}{|c|c|c|c|c|c|c|c|c|c|}
\hline HPV-positive ${ }^{a}$ & 1278 & 102 & 8.0 & 6.6 & 9.6 & 154 & 12.1 & 10.4 & 14.0 \\
\hline HPV16 & 201 & 40 & 19.9 & 15.0 & 26.1 & 49 & 24.4 & 19.0 & 30.9 \\
\hline HPV18 & 74 & 8 & 10.8 & 5.6 & 20.5 & 12 & 16.2 & 9.6 & 26.8 \\
\hline
\end{tabular}

\section{RESULTS}

Testing Results

HPV genotype and cytology counts are given for all HPV-positive women, overall and stratified by median age (Table 1). Categorising HPV-positive women hierarchically based on genotype, 650 (20.8\%) women were HPV16 positive, 221 (7.1\%) were HPV18 positive, and 2148 (69.0\%) were positive for other high-risk HPV; and 92 (3.0\%) for whom no HPV genotype details were recorded; these were included in the overall HPV positive category. There was an increasing percentage of HPV-positive women with highgrade cytology with increasing cancer risk allocated to the individual HPV genotype $\left(P_{\text {trend }}<0.001\right)$. Older women were more likely to have a NILM cytology than younger women $(P<0.001)$.

The overall cumulative CIN3 + risk after testing HPV-positive, regardless of cytologic interpretation or HPV genotype, was $23.9 \%(95 \% \mathrm{Cl}=22.7-24.3 \% ; n=745)$ and $\mathrm{CIN} 2+$ risk was $30.0 \%$ $(95 \% \mathrm{Cl}=30.1-33.4 \% ; n=964)$ (data not shown).

Risks among HPV-positive women referred immediately to colposcopy (ASC-US or worse cytology)

The CIN3+ risk for HPV-positive women with any abnormal cytology, ASC-US or more severe ( $\geq$ ASC-US) cytology was $38.2 \%$ $(95 \% \mathrm{Cl}=35.8-40.6 \%)$.

CIN3 + and CIN2 + risks for pairwise combinations of HPV and $\geq$ ASC-US categories are shown in Table 2. Women with HPV16positive high-grade cytology were at the highest risk of CIN3+ (75.7\%, $95 \% \mathrm{Cl}=69.4-81.5 \%)$ and $\mathrm{CIN} 2+(81.0 \%, 95 \% \mathrm{Cl}=$ 75.1-86.2\%). By comparison, women with other high-risk HPVpositive ASC-US cytology were at the lowest risk of CIN3+ $(18.1 \%$, $95 \% \mathrm{Cl}=15.0-21.7 \%)$ and $\mathrm{CIN} 2+(27.7 \%, 95 \% \mathrm{Cl}=24.0-31.9 \%)$.

Stratified on the median screening age of 44 years (IQR: 38-52 years), overall CIN3 + risk was higher for younger women (34-43 years) $(30.6 \% ; 95 \% \mathrm{Cl}=28.3-33.2 \% ; n=457)$ compared to older women (44-69 years) $(18.1 \% ; 95 \% \mathrm{Cl}=16.3-20.9 \% ; n=288)(P<$ $0.001)$. Figure 2 show risks of CIN3 + for HPV genotype and highgrade versus low-grade cytology. Within each age group, higher risk HPV and more severe cytologic categories independently increased the CIN3+ risk. Younger women were at higher CIN3+ risk than older women but the differences were only significant for all HPV-positive ASC-US (27.7\%, vs. $16.2 \%, P<0.001)$ and HPV16positive ASC-US $(47.8 \%$, vs $29.2 \%, P=0.02)$ and LSIL $(35.2 \%$, vs. $26.1 \%, P=0.01$ ) (data not shown).

Risks among HPV-positive women with NILM cytology Women positive for HPV16/18 with NILM cytology had higher CIN3 + risk compared to women positive for other high-risk HPV with NILM cytology. CIN3+ risk by HPV genotype for NILM were $19.9 \%(95 \% \mathrm{Cl}=15.0-26.1 \%)$ for HPV $16,10.8 \%(95 \% \mathrm{Cl}=5.6-$ $20.5 \%)$ for HPV18 and $5.5 \%(95 \% \mathrm{Cl}=4.2-7.1 \%)$ for other high-risk HPV (Table 3).
Among those women with HPV-positive and NILM cytology results, 626 (49.0\%) re-tested HPV positive in a year. Among those who repeatedly tested HPV-positive, most (539 of 564, 95.6\%) were positive for the same HPV genotype. Twelve-month CIN3+ risks were $29.5 \%(95 \% \mathrm{Cl}=21.8-39.3 \%)$ among women who twice tested HPV16 positive, $15.6 \%(95 \% \mathrm{Cl}=6.8-33.5 \%)$ among women who twice tested HPV18 positive, and $10.2 \%(95 \% \mathrm{Cl}=7.7-13.6 \%)$ among women who twice tested positive for other high-risk HPV (Table 4). By comparison, the CIN3 + risk for twice testing high-risk HPV positive, regardless of specific HPV results, was $14.0 \%(95 \%$ $\mathrm{Cl}=11.4-17.2 \%)$.

Among women with an HPV-positive/NILM result at baseline, those aged 34-43 years had a higher CIN3 + risk overall (11.9\%; 95\% $\mathrm{Cl}=9.1-14.0 \%)$ than women aged $44-69$ years $(4.8 \% ; 95 \% \mathrm{Cl}=$ 3.4-6.7\%) $(P<0.001)$ (Figure 3 and Supplementary Table 1). By HPV genotype, the risk between the two age groups were different for other high-risk HPV only $(P<0.001)$. When stratified for age, the results among HPV genotypes upon second HPV testing also were only significantly different among women who were overall HPV positive and women $(P<0.001)$ who were other high-risk HPV positive $(P<0.001)$. All other persistent HPV genotypes had $P$-values greater than 0.05 (data not shown).

\section{DISCUSSION}

For combinations of HPV-genotype and cytology results using real-world screening data in Norway, women who tested positive for HPV16 and/or HPV18 genotype had a higher risk of CIN3+ and CIN2+ compared with women who tested positive for other highrisk. This was found across every cytologic result. Women, who tested positive for HPV16 and/or HPV18 and $\geq$ ASC-US had a higher risk for $\mathrm{CIN} 3+$ and $\mathrm{CIN} 2+$, and should be referred for immediate colposcopy. The benefits and harms of managing women based on HPV positivity and cytology results can be better balanced by managing women with other high-risk HPV (not HPV16 or HPV18) and low-grade cytology (ASC-US or LSIL) with surveillance.

In Norway, cervical cancer management has been based on results from cervical cytology primary screening. ${ }^{28}$ The switch to the more sensitive HPV test as the primary screening test can lead to over-referral to colposcopy, again leading to increased likelihood of morbidity. ${ }^{43-45}$ Although an HPV positive result alone does confer a high risk of cervical precancer and cancer compared to a negative result, not all HPV genotypes are equally likely to persist ${ }^{46}$ or develop into precancer or cancer. ${ }^{10}$

To increase the accuracy of the HPV screening, a cytological evaluation of HPV positives with divergent clinical management at threshold ASC-US+ has been recommended internationally. Although the Bethesda classification provide criteria for cytological definitions universally, systematic differences between 


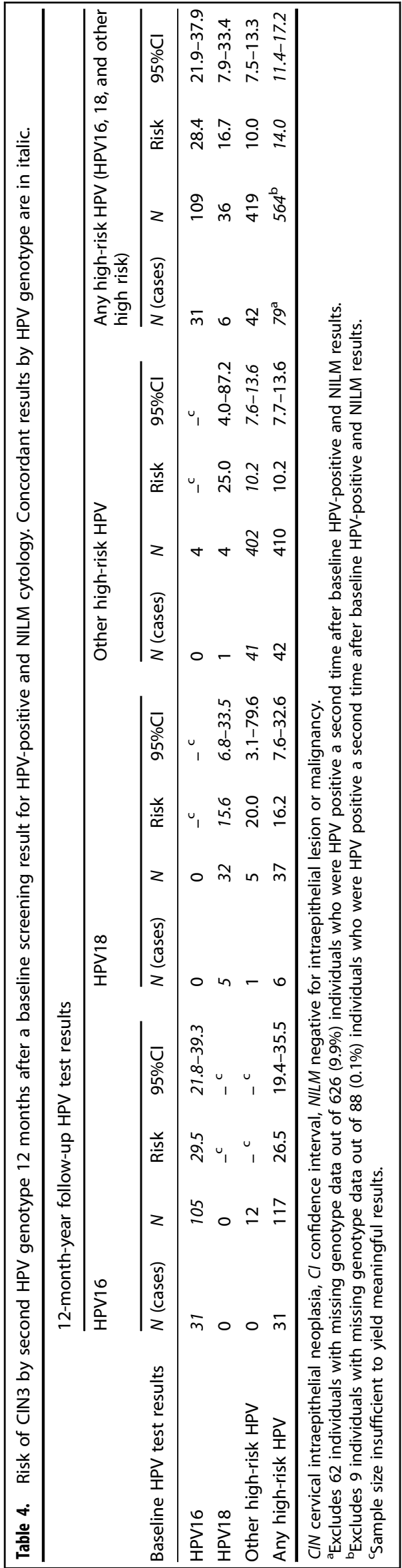

pathology reviewers and countries has been reported in replicating cytological results. ${ }^{47}$ These differences might explain variation in reported proportion of those with ASC-US+ cytology (range 27-46\%). ${ }^{48-50}$ Inter-laboratory differences for cytology results, are commonly observed in Norway, suggesting further that locally agreed diagnostic criteria and routines are applied. ${ }^{51}$ HPV testing in screening, however, demonstrated an excellent agreement between labs, ${ }^{24}$ suggesting wider application of molecular testing in the follow-up. Our study demonstrate that HPV genotyping provides further risk stratification and reduces an over-referral of all women who have an HPV positive and low-grade or NILM cytology result to colposcopy and biopsy.

To avoid an over-referral of all women who have an HPV positive and low-grade or NILM cytology result to colposcopy and biopsy, HPV genotyping provides further risk stratification.

The present analysis of HPV genotypes show consistent results with the POBASCAM and ATHENA trials, which showed higher precancer and cancer risk among HPV16/18 positive women (without cytology testing). ${ }^{34,52,53}$ With cytology testing included, the Portland-Kaiser study, a large cohort of $\sim 20,000$ women, found that HPV16 had a 2.7 times higher risk of precancer and cancer compared to other HPV genotypes across all cytology results. ${ }^{54}$

Among women with baseline cytology of NILM and an HPV genotype of HPV16, 18, or other high-risk HPV type, women who continue to test positive for the same HPV genotype upon two screening rounds had an elevated risk compared to those with whole sub-cohort of women with HPV-positive and NILM cytology result at baseline. CIN3+ risk was highest for women with persistent HPV16 positive results (30\%) and lowest for persistent other high-risk HPV-positive results (10\%), indicating that the most of the $14 \%$ CIN3 + risk for women with NILM cytology and repeat HPV-positive test is due to persistent HPV16 infection. Similarly, an English pilot study found that HPV 16/18 triage of persistently high-risk HPV-positive and cytological negative women 12 months after primary screening added very little in terms of a clinical benefit such as additional detection of CIN2 + after baseline HPV genotyping. ${ }^{55}$ It must be noted that, in this analysis, sample sizes among women testing for two different HPV genotypes upon repeat testing were small [i.e. HPV18 followed up by other highrisk HPV positivity $(n=4)$ ]. Thus, these findings require replication in larger populations.

The conclusions drawn from the genotype results for the Norwegian HPV screening implementation provide the basis for "equal management for equal benchmark risk" approach as performed in the US and certain European countries. This riskbased approach ${ }^{12,56}$ uses a screening (HPV genotype and cytology) result as a benchmark for clinical management decisions for HPV-positive women. Based on real-world screening results from the NCCSP, the risk threshold for a colposcopy referral among HPV-positive women has been equivalent to the risk for other high-risk HPV and LSIL result or $\sim 37.3 \%$ for CIN2+ and $24.4 \%$ for $\mathrm{CIN} 3+$. Women above this threshold, such as those with high-grade cytologic abnormalities or low-grade cytologic abnormalities and test HPV16 or HPV18 positive, should immediately be referred to colposcopy and biopsy. Women with one of these risk markers, other high-risk HPV positive with low-grade cytologic abnormalities or test HPV16 or HPV18 positive with NILM have a risk for $\mathrm{CIN} 3+$ below $24.4 \%$ and can be re-tested in 12 months. Women with neither marker, i.e., NILM cytology and positive for other high-risk HPV, can be retested in 24 months as they have less than half the precancer and cancer risk of women with one of these risk markers.

This analysis focuses on immediate risks of CIN3+ by HPV genotype, including results for women with HPV+ and NILM cytology on their baseline test that are retested after one year. The negative predictive value of this follow-up regimen can be confirmed in long-term follow-up only. The relative rank order between the risk groups observed in this study is consistent with 
a

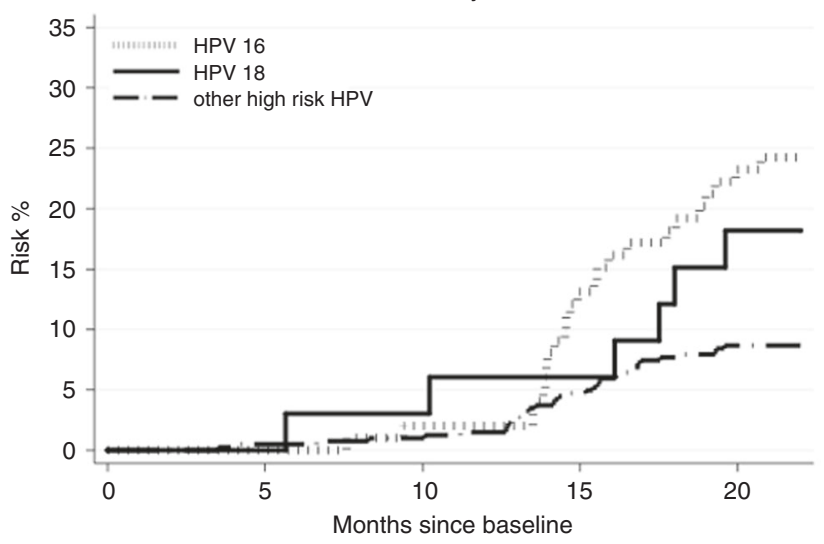

b

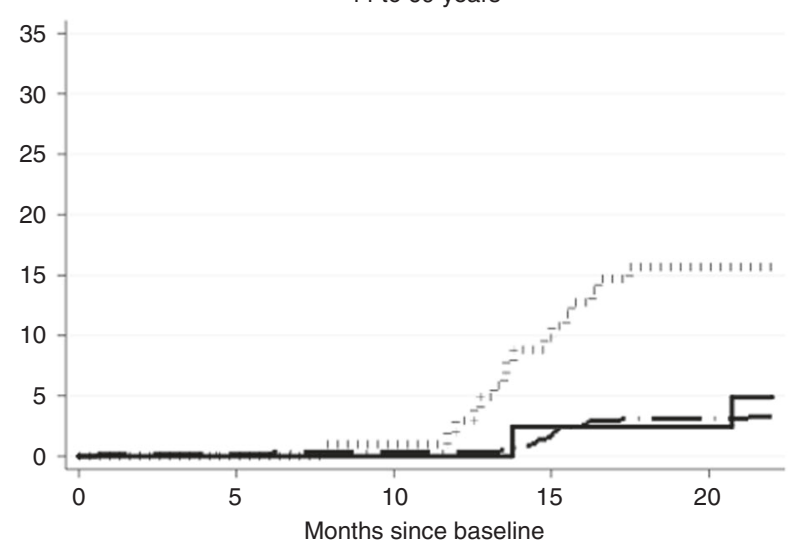

Fig. 3 Risk of CIN3 + by NILM cytology stratified by genotype. (a) 34-43-year-old women (b) 44-69-year-old women. Abbreviations: NILM, negative for intraepithelial lesion or malignancy. Other high-risk HPV includes 31, 33, 35, 39, 45, 51, 52, 56, 58, 59, 66, 68.

those HPV genotype/cytology screening results reported elsewhere. ${ }^{12,56}$ It must also be noted that the threshold at which the risk of cancer in an individual patient outweighs any disadvantage of management and treatment varies according to the patient's characteristics and local service considerations. In a country like Norway, with an established screening program and with high follow-up of screen positives, the threshold for colposcopy referral and treatment may be set higher safely, due to the relatively low risk among women with negative test results. Taking these factors under consideration, these results should be interpreted as a basis for the incorporation of HPV genotyping into primary screening as well as a rationale for risk reductions following over-referral of women to colposcopy and biopsy after primary HPV screening using real-world screening data.

There were several limitations. First, there was only a short followup time for this cohort. Second, estimates of cervical disease (CIN2+ and $\mathrm{CIN3}_{+}$) were calculated based on women who underwent concurrent colposcopy and biopsy. Women with an NILM cytology and HPV-negative result are not typically referred to colposcopy and biopsy and therefore, their cervical histology status is not known. Women who do undergo immediate colposcopy and biopsy with an NILM cytology result, which is against NCCSP and most other cervical cancer screening guidelines, likely represent a biased sample. ${ }^{57}$ It has also been shown that precancer and cancer risk for women with an NILM cytology and HPV-positive result is 5.9\%, making the likelihood of undetected precancer and cancer in a subset of these women quite low. ${ }^{49}$

Despite these limitations, this study has notable strengths. This is the first study to investigate the role of HPV partial genotyping and cytology in an HPV-based screening programme in Norway. Norway has a population-based registration system of cytology results and biopsies and all results are included even if women chose to attend private healthcare. This system reduces loss to follow-up risk and selection biases and has shown to be highly reproducible. $^{58}$ Further, data were collected uniformly, and laboratory tests were performed within an organised cervical cancer screening program with relatively high-population coverage. Results of this analysis can, therefore, be generalisable to a larger population-based cervical screening program, which is a prerequisite for advocating population-wide cancer screening management by HPV genotype. While not generalisable to certain other populations with different patterns of care, compliance, testing, rescreening intervals, or risk factors, ${ }^{12}$ the results of this study have altered the management protocol for HPV-positive women living in Norway, ${ }^{59}$ providing an example of using population-based screening data as a basis for which to implement screening (Supplementary Fig. 2). The results obtained are thus valuable for both Norwegian and non-Norwegian policymakers, as well as for public health organisations that aim to reduce cervical cancer burden.

In conclusion, we found that HPV16 and HPV18 have a higher risk for $\mathrm{CIN} 3+$ and $\mathrm{CIN2}+$ and partial HPV genotyping, in combination with cytology results, can better differentiate HPVpositive women at higher and lower risk for cervical precancer or cancer than cytology alone. This is crucial for women who are at too high risk for surveillance alone, such as those with HPV16/18 and low-grade cytology, while limiting unnecessary colposcopy and biopsy for women with lower precancer or cancer risk, such as those positive for other high-risk HPV genotypes and low-grade cytology. These findings provided the evidence for and adoption of the use of partial HPV genotyping for the management of HPVpositive women aged $34-69$ years in Norway. ${ }^{59}$

\section{AUTHOR CONTRIBUTIONS}

Study design: D.H. and M.N. Data management: G.B.S. Statistical analysis: D.H. Drafted the paper: D.H. Commented on the draft: P.E.C., M.N, B.E., G.B.S., T.B. and A.T. Decision to submit: all authors.

\section{ADDITIONAL INFORMATION}

Ethics approval and consent to participate This study was approved by the Regional Committee for Medical and Health Research Ethics (REK) in South East Norway (REK reference number 2017/1910). REK has given exemption from the consent requirement pursuant to the Norwegian Health Research Act $\S 35$. This exemption is provided that the research in question is of significant interest to society, and the welfare and integrity of the participants is ensured. The specific purpose of this project is to improve cervical cancer prevention and diagnose the disease earlier. The study was performed in accordance with the Declaration of Helsinki.

Consent to publish Not applicable.

Data availability All data not included in this published article are available upon reasonable request from the Cancer Registry of Norway.

Competing interests P.E.C. has received cervical screening tests and diagnostics from Roche, Becton Dickinson, Cepheid, and Arbor Vita Corporation at a reduced or no cost for research. All other authors report no competing interests.

Funding information This work is supported by a grant from the Norwegian Cancer Society, project ID: \#564905. Sponsors had no role in study design, data collection, analysis, and interpretation of data; in the writing of the report or the decision to submit the article for publication. 
Supplementary information is available for this paper at https://doi.org/10.1038/ s41416-020-0790-1.

Publisher's note Springer Nature remains neutral with regard to jurisdictional claims in published maps and institutional affiliations.

\section{REFERENCES}

1. Castle, P. E., Rodriguez, A. C., Burk, R. D., Herrero, R., Wacholder, S., Alfaro, M. et al. Short term persistence of human papillomavirus and risk of cervical precancer and cancer: population based cohort study. BMJ 339, b2569 (2009).

2. Kjaer, S. K., Frederiksen, K., Munk, C. \& Iftner, T. Long-term absolute risk of cervical intraepithelial neoplasia grade 3 or worse following human papillomavirus infection: role of persistence. J. Natl Cancer Inst. 102, 1478-1488 (2010).

3. Vesco, K. K., Whitlock, E. P., Eder, M., Burda, B. U., Senger, C. A. \& Lutz, K. Risk factors and other epidemiologic considerations for cervical cancer screening: a narrative review for the U.S. Preventive Services Task Force. Ann. Intern. Med. 155, 698-705 (2011).

4. Ronco, G., Dillner, J., Elfstrom, K. M., Tunesi, S., Snijders, P. J., Arbyn, M. et al. Efficacy of HPV-based screening for prevention of invasive cervical cancer: followup of four European randomised controlled trials. Lancet 383, 524-532 (2014).

5. Arbyn, M., Ronco, G., Anttila, A., Meijer, C. J., Poljak, M., Ogilvie, G. et al. Evidence regarding human papillomavirus testing in secondary prevention of cervical cancer. Vaccine 30, F88-F99 (2012).

6. Whitlock, E. P., Vesco, K. K., Eder, M., Lin, J. S., Senger, C. A. \& Burda, B. U. Liquidbased cytology and human papillomavirus testing to screen for cervical cancer: a systematic review for the U.S. Preventive Services Task Force. Ann. Intern. Med. 155, 687-697 (2011).

7. von Karsa, L., Arbyn, M., De Vuyst, H., Dillner, J., Dillner, L., Franceschi, S. et al. European guidelines for quality assurance in cervical cancer screening. Summary of the supplements on HPV screening and vaccination. Papillomavirus Res. 1, 22-31 (2015).

8. Naucler, P., Ryd, W., Tornberg, S., Strand, A., Wadell, G., Elfgren, K. et al. Human papillomavirus and Papanicolaou tests to screen for cervical cancer. N. Engl. J. Med. 357, 1589-1597 (2007).

9. Castle, P. E. \& Katki, H. A. Benefits and risks of HPV testing in cervical cancer screening. Lancet Oncol. 11, 214-215 (2010).

10. Wentzensen, N., Schiffman, M., Palmer, T. \& Arbyn, M. Triage of HPV positive women in cervical cancer screening. J. Clin. Virol. 76, S49-s55 (2016).

11. Velentzis, L. S., Caruana, M., Simms, K. T., Lew, J. B., Shi, J. F., Saville, M. et al. How will transitioning from cytology to HPV testing change the balance between the benefits and harms of cervical cancer screening? Estimates of the impact on cervical cancer, treatment rates and adverse obstetric outcomes in Australia, a high vaccination coverage country. Int. J. Cancer 141, 2410-2422 (2017).

12. Katki, H. A., Schiffman, M., Castle, P. E., Fetterman, B., Poitras, N. E., Lorey, T. et al. Benchmarking $\mathrm{CIN} 3+$ risk as the basis for incorporating HPV and Pap cotesting into cervical screening and management guidelines. J. Low. Genit. Trac. Dis. 17, S28 (2013).

13. Wright, T. C. Jr., Stoler, M. H., Sharma, A., Zhang, G., Behrens, C., Wright, T. L. et al. Evaluation of HPV-16 and HPV-18 genotyping for the triage of women with highrisk HPV+ cytology-negative results. Am. J. Clin. Pathol. 136, 578-586 (2011).

14. Wheeler, C. M., Hunt, W. C., Schiffman, M. \& Castle, P. E. Human papillomavirus genotypes and the cumulative 2-year risk of cervical precancer. J. Infect. Dis. 194, 1291-1299 (2006).

15. Castle, P. E., Solomon, D., Schiffman, M. \& Wheeler, C. M. Human papillomavirus type 16 infections and 2-year absolute risk of cervical precancer in women with equivocal or mild cytologic abnormalities. J. Natl Cancer Inst. 97, 1066-1071 (2005).

16. Saslow, D., Solomon, D., Lawson, H. W., Killackey, M., Kulasingam, S. L., Cain, J. et al. American Cancer Society, American Society for Colposcopy and Cervical Pathology, and American Society for Clinical Pathology screening guidelines for the prevention and early detection of cervical cancer. CA Cancer J. Clin. 62, 147-172 (2012).

17. Castle, P. E., Cuzick, J., Stoler, M. H., Wright, T. C. Jr., Reid, J. L., Dockter, J. et al. Detection of human papillomavirus 16, 18, and 45 in women with ASC-US cytology and the risk of cervical precancer: results from the CLEAR HPV study. Am. J. Clin. Pathol. 143, 160-167 (2015).

18. de Sanjose, S., Quint, W. G., Alemany, L., Geraets, D. T., Klaustermeier, J. E., Lloveras, B. et al. Human papillomavirus genotype attribution in invasive cervical cancer: a retrospective cross-sectional worldwide study. Lancet Oncol. 11, 1048-1056 (2010).

19. Khan, M. J., Castle, P. E., Lorincz, A. T., Wacholder, S., Sherman, M., Scott, D. R. et al. The elevated 10-year risk of cervical precancer and cancer in women with human papillomavirus (HPV) type 16 or 18 and the possible utility of type-specific HPV testing in clinical practice. J. Natl Cancer Inst. 97, 1072-1079 (2005).

20. Hosaka, M., Fujita, H., Hanley, S. J., Sasaki, T., Shirakawa, Y., Abiko, M. et al. Incidence risk of cervical intraepithelial neoplasia 3 or more severe lesions is a function of human papillomavirus genotypes and severity of cytological and histological abnormalities in adult Japanese women. Int. J. Cancer 132, 327-334 (2013).

21. Wright, T. C. Jr., Stoler, M. H., Behrens, C. M., Apple, R., Derion, T. \& Wright, T. L. The ATHENA human papillomavirus study: design, methods, and baseline results. Am. J. Obstet. Gynecol. 206, 46.e1-.e11 (2012).

22. Polman, N. J., Veldhuijzen, N. J., Heideman, D. A. M., Snijders, P. J. F., Meijer, C. \& Berkhof, J. Management of HPV-positive women in cervical screening using results from two consecutive screening rounds. International journal of cancer. Int. J. Cancer 144, 2339-2346 (2019).

23. Arbyn, M., Xu, L., Verdoodt, F., Cuzick, J., Szarewski, A., Belinson, J. L. et al. Genotyping for human papillomavirus types 16 and 18 in women with minor cervical lesions: a systematic review and meta-analysis. Ann. Intern. Med. 166, 118-127 (2017).

24. Engesaeter, B., van Diermen Hidle, B., Hansen, M., Moltu, P., Staby, K. M., Borchgrevink-Persen, $\mathrm{S}$. et al. Quality assurance of human papillomavirus (HPV) testing in the implementation of HPV primary screening in Norway: an inter-laboratory reproducibility study. BMC Infect. Dis. 16, 698 (2016).

25. Laboratories A. Realtime High Risk HPV [Website]. https://www.molecular.abbott/ int/en/products/infectious-disease/realtime-high-risk-hpv (Abbott, Abbott Park, Illinois, 2018).

26. Diagnostics R. M. cobas $^{\circledR}$ HPV 2018. https://molecular.roche.com/assays/cobashpv-test/ (2018).

27. Solomon, D. \& Nayar, R. The Bethesda System for reporting cervical cytology: definitions, criteria, and explanatory notes. 2 ed. (Springer-Verlag New-York, Inc., New-York, 2004).

28. Trope A., Engesaeter B., Nygard M., Andreassen T., Lonnberg S. \& Ursin G. [Safe implementation of HPV testing in the Norwegian Cervical Cancer Screening Programme]. Tidsskrift for den Norske laegeforening : tidsskrift for praktisk medicin, ny raekke. 137, (2017).

29. Leinonen, M. K., Hansen, S. A., Skare, G. B., Skaaret, I. B., Silva, M., Johannesen, T. B. et al. Low proportion of unreported cervical treatments in the cancer registry of Norway between 1998 and 2013. Acta Oncologica 57, 1663-1670 (2018).

30. Larsen, I. K., Smastuen, M., Johannesen, T. B., Langmark, F., Parkin, D. M., Bray, F. et al. Data quality at the Cancer Registry of Norway: an overview of comparability, completeness, validity and timeliness. Eur. J. Cancer 45, 1218-1231 (2009).

31. Nygard, J. F., Skare, G. B. \& Thoresen, S. O. The cervical cancer screening programme in Norway, 1992-2000: changes in Pap smear coverage and incidence of cervical cancer. J. Med. Screen. 9, 86-91 (2002).

32. Tavassoli, F. A., Devilee, P. Pathology and genetics of tumours of the breast and female genital organs. Lyon: IARC Press; 2003.

33. Schiffman, M., Hyun, N., Raine-Bennett, T. R., Katki, H., Fetterman, B., Gage, J. C. et al. A cohort study of cervical screening using partial HPV typing and cytology triage. Int. J. Cancer 139, 2606-2615 (2016).

34. Castle, P. E., Stoler, M. H., Wright, T. C. Jr., Sharma, A., Wright, T. L. \& Behrens, C. M. Performance of carcinogenic human papillomavirus (HPV) testing and HPV16 or HPV18 genotyping for cervical cancer screening of women aged 25 years and older: a subanalysis of the ATHENA study. Lancet Oncol. 12, 880-890 (2011).

35. Stoler, M. H., Wright, T. C. Jr., Sharma, A., Apple, R., Gutekunst, K. \& Wright, T. L. High-risk human papillomavirus testing in women with ASC-US cytology: results from the ATHENA HPV study. Am. J. Clin. Pathol. 135, 468-475 (2011).

36. Wentzensen, N., Wilson, L. E., Wheeler, C. M., Carreon, J. D., Gravitt, P. E., Schiffman, M. et al. Hierarchical clustering of human papilloma virus genotype patterns in the ASCUS-LSIL triage study. Cancer Res. 70, 8578-8586 (2010).

37. Castle, P. E., Stoler, M. H., Solomon, D. \& Schiffman, M. The relationship of community biopsy-diagnosed cervical intraepithelial neoplasia grade 2 to the quality control pathology-reviewed diagnoses: an ALTS report. Am. J. Clin. Pathol. 127, 805-815 (2007)

38. Stoler, M. H. \& Schiffman, M. Atypical Squamous Cells of Undetermined Significance-Low-grade Squamous Intraepithelial Lesion Triage Study (ALTS) Group. Interobserver reproducibility of cervical cytologic and histologic interpretations: realistic estimates from the ASCUS-LSIL Triage Study. JAMA 285, 1500-1505 (2001)

39. Castle, P. E., Schiffman, M., Wheeler, C. M. \& Solomon, D. Evidence for frequent regression of cervical intraepithelial neoplasia-grade 2. Obstet. Gynecol. 113, 18-25 (2009).

40. Moscicki, A. B., Ma, Y., Wibbelsman, C., Darragh, T. M., Powers, A., Farhat, S. et al. Rate of and risks for regression of cervical intraepithelial neoplasia 2 in adolescents and young women. Obstet. Gynecol. 116, 1373-1380 (2010).

41. Kaplan, E. L. \& Meier, P. Nonparametric estimation from incomplete observations. J. Am. Stat. Assoc. 53, 457-481 (1958). 
42. Marubini E. \& Valsecchi M. G. Analysing Survival Data from Clinical Trials and Observational Studies (John Wiley \& Sons, 2004).

43. Huh, W. K., Sideri, M., Stoler, M., Zhang, G., Feldman, R. \& Behrens, C. M. Relevance of random biopsy at the transformation zone when colposcopy is negative. Obstet. Gynecol. 124, 670-678 (2014).

44. Arbyn, M., Kyrgiou, M., Simoens, C., Raifu, A. O., Koliopoulos, G., Martin-Hirsch, P. et al. Perinatal mortality and other severe adverse pregnancy outcomes associated with treatment of cervical intraepithelial neoplasia: meta-analysis. Bmj 337 a1284 (2008).

45. Kyrgiou, M., Athanasiou, A., Paraskevaidi, M., Mitra, A., Kalliala, I., Martin-Hirsch, P. et al. Adverse obstetric outcomes after local treatment for cervical preinvasive and early invasive disease according to cone depth: systematic review and metaanalysis. Bmj 354, i3633 (2016).

46. Sand, F. L., Munk, C., Frederiksen, K., Junge, J., Iftner, T., Dehlendorff, C. et al. Risk of CIN3 or worse with persistence of 13 individual oncogenic HPV types. International journal of cancer. Int. J. Cancer 144, 1975-1982 (2018).

47. Scott, D. R., Hagmar, B., Maddox, P., Hjerpe, A., Dillner, J., Cuzick, J. et al. Use of human papillomavirus DNA testing to compare equivocal cervical cytologic interpretations in the United States, Scandinavia, and the United Kingdom. Cancer 96, 14-20 (2002).

48. Demarco, M., Lorey, T. S., Fetterman, B., Cheung, L. C., Guido, R. S., Wentzensen, N. et al. Risks of CIN 2+, CIN 3+, and Cancer by Cytology and Human Papillomavirus Status: The Foundation of Risk-Based Cervical Screening Guidelines. J. Low. Genit. Trac. Dis. 21, 261-267 (2017).

49. Katki, H. A., Kinney, W. K., Fetterman, B., Lorey, T., Poitras, N. E., Cheung, L. et al. Cervical cancer risk for women undergoing concurrent testing for human papillomavirus and cervical cytology: a population-based study in routine clinical practice. Lancet Oncol. 12, 663-672 (2011).

50. Wright, T. C. Jr., Behrens, C. M., Ranger-Moore, J., Rehm, S., Sharma, A., Stoler, M. H. et al. Triaging HPV-positive women with p16/Ki-67 dual-stained cytology: Results from a sub-study nested into the ATHENA trial. Gynecologic Oncol. 144, 51-56 (2017).

51. Skare, G. B., Bjørge, T. \& Tropé, A. Årsrapport 2016, Livmorhalsprogrammet (2016).

52. Dijkstra, M. G., van Niekerk, D., Rijkaart, D. C., van Kemenade, F. J., Heideman, D. A., Snijders, P. J. et al. Primary hrHPV DNA testing in cervical cancer screening: how to manage screen-positive women? A POBASCAM trial substudy. Cancer Epidemiol. Biomarkers Prev. 23, 55-63 (2014).

53. Rijkaart, D. C., Berkhof, J., van Kemenade, F. J., Coupe, V. M. H., Hesselink, A. T., Rozendaal, L. et al. Evaluation of 14 triage strategies for HPV DNA-positive women in population-based cervical screening. Int. J. cancer 130, 602-610 (2012).
54. Schiffman, M., Glass, A. G., Wentzensen, N., Rush, B. B., Castle, P. E., Scott, D. R. et al. A long-term prospective study of type-specific human papillomavirus infection and risk of cervical neoplasia among 20,000 women in the Portland Kaiser Cohort Study. Cancer Epidemiol. Biomarkers Prev. 20, 1398-1409 (2011).

55. Rebolj M., Brentnall A. R., Mathews C., Denton K., Holbrook M., Levine T. et al. 16/ 18 genotyping in triage of persistent human papillomavirus infections with negative cytology in the English cervical screening pilot. Br. J. Cancer 121 455-463 (2019).

56. Castle, P. E., Sideri, M., Jeronimo, J., Solomon, D. \& Schiffman, M. Risk assessment to guide the prevention of cervical cancer. J. Low. Genit. Trac. Dis. 12, 1-7 (2008).

57. Canfell, K., Caruana, M., Gebski, V., Darlington-Brown, J., Heley, S., Brotherton, J. et al. Cervical screening with primary HPV testing or cytology in a population of women in which those aged 33 years or younger had previously been offered HPV vaccination: Results of the Compass pilot randomised trial. PLoS Med. 14 e1002388 (2017).

58. Lonnberg, S., Leinonen, M., Malila, N. \& Anttila, A. Validation of histological diagnoses in a national cervical screening register. Acta Oncologica 51, 37-44 (2012).

59. NCCSP NCCSP. HPV i primærscreening. https://www.kreftregisteret.no/en/ screening/Cervical-Cancer-Screening-Programme/Helsepersonell/screeningstrategiog-nasjonale-retningslinjer/hpv-i-primarscreening/ (Cancer Registry of Norway, Oslo, Norway, 2018).

(i) Open Access This article is licensed under a Creative Commons Attribution 4.0 International License, which permits use, sharing, adaptation, distribution and reproduction in any medium or format, as long as you give appropriate credit to the original author(s) and the source, provide a link to the Creative Commons license, and indicate if changes were made. The images or other third party material in this article are included in the article's Creative Commons license, unless indicated otherwise in a credit line to the material. If material is not included in the article's Creative Commons license and your intended use is not permitted by statutory regulation or exceeds the permitted use, you will need to obtain permission directly from the copyright holder. To view a copy of this license, visit http://creativecommons. org/licenses/by/4.0/.

(c) The Author(s) 2020 